\title{
Duration judgments of naturalistic events in the auditory and visual modalities
}

\author{
MARILYN G. BOLTZ \\ Haverford College, Haverford, Pennsylvania
}

\begin{abstract}
Two experiments were performed to examine whether the same underlying mechanisms apply to the duration estimates of both auditory and visual events. In Experiment 1, it was found that the durations of visual scenes are reproduced with the same level of accuracy in prospective and retrospective situations when these display a predictable array of information, a result consistent with past research on auditory durations. Experiment 2 further revealed that when participants are asked to prospectively or retrospectively judge the durations of various naturalistic events in their auditory, visual, or audiovisual modality, no differences in either accuracy or bias are observed. These findings diverge from previous research and are argued to stem from different processing mechanisms that arise from naturalistic events.
\end{abstract}

Duration judgments are implicit in many everyday activities and are useful for ensuring that time is used efficiently for scheduling purposes. Many of the events we routinely encounter are multimodal in nature and offer information in both the visual and the auditory modalities. For example, suppose that a witness to a violent argument is later asked to estimate its duration. Depending on the witness's distance and location, he or she may have focused primarily on the visual gestures and actions or on the exchange of words and voices. The question addressed here is whether attending to one modality or the other results in more accurate estimates of both experienced and remembered duration. In addition, does time estimation ability depend on the inherent structure of the events to be judged?

\section{Temporal Processing in the Auditory Versus Visual Modalities}

These questions relate to more fundamental issues concerning how different perceptual systems compare in their processing abilities. One common technique is to present participants with conflicting information from different modalities to determine whether one captures the other. A typical finding in such experiments is that of visual dominance (Posner, Nissen, \& Klein, 1976). In ventriloquism, for example, speech produced with no visible facial movements from a human performer appears to emanate from the moving lips of a puppet (Bertelson, 1999). Several individuals (e.g., Kubovy, 1988; Welch, 1999; Welch \& Warren, 1980) have noted that the particular modality that dominates is that which is more

This research was supported by a Faculty Research Grant from Haverford College. The author thanks Erika Fulton for the collection of data. Correspondence concerning this article should be addressed to M. G. Boltz, Department of Psychology, Haverford College, Haverford, PA 19041 (e-mail: mboltz@haverford.edu). precise and appropriate for the source of conflict. For example, the visual modality dominates in the case of ventriloquism, because is it more trustworthy for spatial location. Conversely, audition has been found to dominate when there are temporal discrepancies between modalities. This is illustrated by the phenomenon of $a u$ ditory driving, in which a sequence of repetitive visual stimuli, unfolding at a constant tempo, is perceived to increase in rate when accompanied by an accelerating sequence of auditory clicks. No such misperceptions arise, however, when participants are asked to judge the rate of auditory clicks in the presence of visual sequences that vary in their tempo (Gebhard \& Mowbray, 1959; Shipley, 1964).

Similar conclusions have been offered by others who have assessed performance on tasks in which the modality of stimulus presentation has been varied. Although some have disputed this claim (e.g., Handel, 1988), many have argued that the visual modality is better at processing spatial information, whereas the auditory modality is more sensitive to temporal information - a view that Glenberg and Swanson (1986) have termed the temporal distinctiveness theory. In support, Metcalfe, Glavanov, and Murdock (1981) reported that auditory stimuli are better recalled by temporal order but that visual stimuli are better recalled by spatial location. In the processing of pattern information, auditory rhythms are learned at a faster rate (Handel \& Buffardi, 1968) and are better remembered (Glenberg \& Jona, 1991; Glenberg, Mann, Altman, Forman, \& Procise, 1989) than visual rhythms. In addition, when rhythms are disrupted by random variations of the interstimulus interval (ISI), the remembering of auditory patterns markedly declines, whereas that of visual ones does not (Glenberg \& Jona, 1991).

Given this research, one might expect duration estimates to be more accurate when events are heard rather than seen. The empirical evidence is mixed. Grondin has conducted a number of psychophysical studies and has 
found that auditory signals yield better discrimination performance than do visual ones, a finding that applies to both filled (Grondin, Meilleur-Wells, Ouellette, \& Macar, 1998) and empty (Grondin et al., 1998; Grondin \& Metthé, 1993; Grondin \& Rousseau, 1991) intervals. When biases emerge, auditory sequences are judged to be longer than visual ones (Goldstone \& Goldfarb, 1964; Goldstone \& Lhamon, 1972, 1974; Penney, Allan, Meck, \& Gibbon, 1998; Walker \& Scott, 1981; Wearden, Edwards, Fakhri, \& Percival, 1998). This phenomenon appears to be a very reliable one that has been attributed to uncontrolled differences in stimulus characteristics, such as intensity and movement (Goldstone \& Lhamon, 1974), or to differential rates of an internal clock (Penney et al., 1998; Wearden et al., 1998). As has been noted by Grondin (2001), a higher pulse rate for audition would enhance the definition of a given interval and, thereby, result in more accurate discrimination.

Although all of these types of studies reveal modality differences in duration judgments, other studies do not. Crowder and Green (1987), for example, required participants to compare the durations of word lists that were visually versus auditorily presented and consistently found no effects of stimulus modality. This null effect has been obtained by others with production tasks (Mohan, 1975), as well as with verbal estimation and magnitude estimation tasks (Bobko, Thompson, \& Schiffman, 1977). Such findings have been interpreted as evidence for a centralized mechanism that operates independently of stimulus modality.

In sum, the issue of whether event durations are more accurately judged in the auditory than in the visual modality remains unresolved. Some of these divergent findings are likely due to task and methodological differences. Penney et al. (1998), for example, noted that modality differences are more apt to emerge in within-subjects designs in which auditory and visual intervals are directly compared with one another. In contrast, between-subjects designs often yield null effects. In the present research, an additional set of factors is considered that has not yet been acknowledged - namely, those of judgment paradigm, the ecological validity of experimental stimuli, and learning.

\section{The Potential Impact of Judgment Paradigm and Naturalistic Events}

To date, all of the research in which modality effects on judged duration have been examined has relied exclusively on the prospective paradigm, in which participants know in advance that time judgments will be required. This type of paradigm allows one to assess experienced duration (i.e., time in passing) and is useful for addressing psychophysical issues. However, its methodological demands can also encourage the use of conscious timekeeping strategies (e.g., counting or tapping), which may contribute to the pattern of observed results. In contrast, retrospective designs reduce this concern, because participants do not know that a time judgment will be required until after an event has transpired - meaning that there is no reason to consciously monitor time during the event's initial encoding. In addition to providing greater methodological rigor, retrospective judgments enable one to assess remembered duration estimates, which, although less investigated, are just as common in everyday life as prospective ones. In lieu of consciously monitoring time while performing a given activity, people are often more focused on the what and why of the activity (i.e., nontemporal information) and only later may estimate the event's duration in retrospect. Previous research has found that people are able to remember the duration of various auditory stimuli, such as musical selections (Boltz, 1995, 1998, 1999) and ecological sounds (Boltz, 1992, 1994), and it is worthwhile to determine whether this ability generalizes to visual events.

A second notable feature of past research is that it has tended to rely on relatively artificial stimuli, such as a single tone or an illuminated light. Although such stimuli can provide rigorous experimental control, it is not always clear whether the results of their use can be generalized to everyday behavior. Sights and sounds are more typically encountered in the context of social activities (e.g., a basketball game, driving, or people walking), in which the pattern of auditory information directly corresponds to a visual source. Events such as these are much more familiar - a factor that has been found to influence duration estimates (Kowal, 1976, 1981; Von Sturmer, 1966; Warm, Greenberg, \& Dube, 1969; Witherspoon \& Allan, 1985). In addition, natural events not only are dynamic and unfold over time, but also are structured in time.

Jones and Boltz (1989) have noted that natural event structure can vary in its degree of predictability and have characterized this in terms of a continuum of structural coherence. At one end are highly coherent events that display a high degree of internal organization stemming from multiple sources (see Boltz, 1998, and Boltz, Kupperman, \& Dunne, 1998, for discussions), one of which is event timing, in which an underlying rhythm gives rise to a lawfully recurrent sequence of nontemporal items. Much of Western music, for example, displays this type of structural array (Berry, 1976; Lerdahl \& Jackendoff, 1983; Piston, 1978), as do speech utterances (Goldman-Eisler, 1972; Grosjean, Grosjean, \& Lane, 1979; Martin, 1972) and many ecological sounds (Boltz, 1992). Similarly, within the visual modality, highly learned motor abilities (e.g., walking, riding a bicycle, or driving a car) are performed skillfully, with no hesitations and a smooth coordination of body movements. In each case, the sequence of nontemporal items (i.e., notes, words, pitch frequencies, or body movements) unfolds in a temporally integrated fashion with a characteristic rhythm, rate, and total duration. Both structural dimensions are inextricably entwined and serve to jointly characterize the event, thereby rendering a high degree of internal organization to the event's total time span.

On a behavioral level, highly coherent events facilitate encoding and remembering in a number of different ways. Given a high degree of internal predictability, people are easily able to track an event as it unfolds (Large \& Jones, 1999; Pfordresher, 2003) and to learn its overall structural organization (Boltz, 1991). In addition, the joint relationship between temporal and nontemporal information allows both to be encoded in a unified fashion, particularly 
with greater learning experience, in which the inherent organization of the event will be more apparent. Attending to one dimension leads to an incidental learning of the other, and no performance decrement occurs when both are attended to at once (Boltz, 1998, 1999). This means that duration judgments will be accurate in both prospective and retrospective situations, in which people are focused primarily on temporal and nontemporal information, respectively.

At the other end of the continuum are less coherent events. In these, the structural array is a very unpredictable one, in which the sequence of nontemporal information appears to be ill timed. This is particularly apparent in both the production and the perception of events at early stages of learning. For example, the speech of a foreigner learning a new language or the music of a novice pianist often displays a disjointed rhythm, as do the body movements of an individual learning a new motor skill, such as walking or riding a bicycle. Similarly, a structurally coherent event may not appear as such when it is initially encountered, because one has not yet perceived the lawful arrangement of event structure. Not only will such events be very difficult to attentionally track, but given that temporal and nontemporal information are structurally dissociated from one another, each will require independent processing resources to be encoded into memory. Hence, duration will be well remembered only when it is selectively attended to during encoding (i.e., prospective situations), and conditions of divided attending will lead to decrements in performance. Retrospective paradigms, on the other hand, will yield highly variable and inaccurate estimates, and people may rely on an availability heuristic to infer remembered duration. The latter concept has been borrowed from certain memory-based models of time estimation behavior (Block, 1985; Ornstein, 1969), which assert that remembered duration is inferred from the amount of nontemporal information stored in memory. Given that people are unlikely to encode event duration under retrospective conditions, they therefore rely on the only source of information available in memory - namely, the number of chunks (Ornstein, 1969) or changes (Block, $1985)$ within the array of nontemporal information. The more the chunks or changes that are apparent, the longer the time estimates will be.

Some support for this overall framework comes from a study by Boltz (1999), in which participants were presented with a set of melodies that varied in their internal structure. Some were very lawful (coherent), in that the sequence of notes and melodic accents recurred with a rhythmic periodicity, whereas others were relatively incoherent, in that the array of pitch information appeared with an irregular periodicity. During an initial learning phase, the participants were presented with both types of melodies on two, four, or eight different occasions while rating them on various perceptual dimensions. In addition, selective attending was manipulated, in that the participants were instructed to attend to a melody's pitch sequence, rhythm, or duration or to all three dimensions simultaneously. Afterward, all the participants were asked to perform three types of memory tasks - namely, duration reproduction, rhythm recall, and pitch recognition. Given that some dimensions were attended to, but not others, these subsequent memory tasks were, therefore, prospective or retrospective ones across different attending conditions.

The results showed that after two learning trials, all the melodies, regardless of their internal structure, yielded a comparable pattern of performance by participants on each of the three tasks. The memory of any given structural dimension, including total duration, was quite accurate when it was selectively attended to but very poor when attention was directed elsewhere or divided among other dimensions. With additional learning experience, however, the duration memory of coherent melodies became quite accurate ( $9 \%$ absolute error), regardless of whether the participants had attended to duration, rhythm, or pitch alone during the initial encoding phase. More important, there was no performance decrement when attention was divided among the three structural dimensions. Incoherent melodies, on the other hand, yielded a level of performance that was comparable to that in the two-trial learning condition. Duration was remembered well if it was selectively attended to during encoding, but performance declined markedly when attending was directed toward pitch or rhythm and declined even more when attending was divided among all three dimensions.

In sum, then, these results illustrate that with sufficient learning experience, the temporal and nontemporal information for coherent events are jointly encoded into the cognitive system, so that duration memory is comparable across prospective and retrospective conditions. Conversely, incoherent events reveal evidence for an independent encoding of structural information that is more typical of the results of past research. Although duration judgments are relatively accurate in prospective situations, performance decrements arise in retrospective situations and/or conditions of concurrent processing (Brown, 1985; Brown \& Stubbs, 1992; Fortin, 1999; Macar, Grondin, \& Casini, 1994).

The purpose of the present research is to extend these findings by investigating duration estimates of naturalistic events that occur in the visual and auditory modalities. Experiment 1 was performed to examine whether effects of event structure, learning, and judgment paradigm exert the same influence on visual events as has previously been found for auditory ones. In Experiment 2, the two modalities were directly compared to determine whether prospective and retrospective duration estimates vary when participants are presented with auditory or visual information alone or with both in tandem.

\section{EXPERIMENT 1}

Experiment 1 addressed two main issues. The first was how the learning and remembering of visual durations compare across the prospective and retrospective paradigms. If results from previous research on auditory events (Boltz, 1998, 1999) generalize to the visual domain, the 
degree of accuracy should be equivalent for both types of judgments, and this should be particularly true after an extensive learning experience. This would suggest that for both the auditory and the visual modalities, an event's temporal and nontemporal information is jointly encoded into memory, so that attending to one dimension leads to an incidental learning and remembering of the other. On the other hand, temporal information may be less salient in the overall structural characterization of a visual event, and if so, duration estimates may be accurate only in prospective situations and not in retrospective ones.

The second issue addressed in Experiment 1 involves effects due to event structure. Does structural coherence facilitate the learning and remembering of visual durations, as is true of the auditory domain? The research by Glenberg and Jona (1991) suggests not. In their experiments, they found not only that people are better able to reproduce rhythmic sequences in the auditory modality but also that predictable rhythms are more facilitating for audition than for vision: In the visual modality, predictable rhythms produce a lower level of performance that is comparable to that for unpredictable rhythms. If these results can be generalized to time estimation behavior, one would expect remembered duration judgments to be relatively inaccurate not only for visual events, but also equally so for those that are both coherently and incoherently structured. On the other hand, even though people have more difficulty remembering visual rhythms than auditory ones, it may be that coherent arrangements of temporal/nontemporal information have the same beneficiary impact upon selective attending skills, regardless of event modality. After all, when one watches others walk, type, or play basketball, these relatively familiar events contain structural arrays that are very similar to those of their auditory correlates. Events in both modalities contain an internal arrangement of distinct nontemporal markers that can unfold in a rhythmic or arrhythmic fashion. Given this, a similar pattern of results may occur for both, so that predictably structured (coherent) events are more accurately remembered than less predictable (incoherent) events. Some support for this idea comes from Holleran and Jones (2001), who found that although participants were better able to recall element durations and detect interval changes in coherent than in incoherent sequences, these effects were comparable across the auditory and visual modalities.

Experiment 1 relied on an experimental design that was very similar to that in Boltz (1999), which assessed the remembering of auditory durations. During an initial learning phase, participants were presented with six visual events and were told to attend either to their total durations (prospective instructions) or to the sequences of actions (retrospective instructions) while rating them on several perceptual dimensions. Half of the participants encountered each event on four occasions, whereas the remainder of the participants received more learning experience by seeing each event on eight occasions. The last experimental manipulation involved event structure: Two of the events were highly predictable in nature and displayed an invariant periodicity between successive markers (e.g., dribbling by an expert basketball player); two were relatively unpredictable in that the interitem periodicity was more variable (e.g., the staggering footsteps of a drunk); and lastly, two events displayed an intermediate degree of predictability, in that the total time span was filled with a continuous array of information with no structural markers (e.g., the flight of a Frisbee). After performing the set of rating tasks for their respective number of learning trials, all the participants were asked to reproduce the duration of each event through a buttonpress.

\section{Method}

Design and Participants. The design was a $3 \times 2 \times 2$ mixed factorial. All the participants viewed a set of visual scenes in which the characters' actions varied in their internal structure (coherent, incoherent, or continuous). The between-subjects factors were the number of exposures to each event during the initial learning phase (four or eight), and the type of instructions administered (prospective or retrospective)

Ninety-six students from an introductory psychology course at Haverford College participated in the experiment for course credit. Each had normal (or corrected) vision.

Stimulus materials. A total of 12 visual scenes, described in Table 1, were staged and filmed onto videotape. Each depicted a college-age male and/or female engaged in a given activity that had a clear, nonarbitrary beginning and end.

The primary dimension in which these different activities varied was that of event structure. Four were termed coherent, in that the temporal periodicity between successive body movements was a relatively invariant one, so that the actor's overall performance appeared to be highly skilled and rhythmic in nature. For purposes of methodological rigor, the same activities were also performed in a relatively incoherent fashion. As is shown in Table 1, the incoherent events exhibited approximately the same number of action steps and total duration as did their respective coherent counterparts. In addition, even though the mean ISI for the various action steps was relatively comparable across the coherent and incoherent versions of a given event, the degree of variability in the ISI was significantly higher in the incoherent version than in the coherent one. In order to quantify this, each visual scene was digitized onto a Macintosh Performa 6115 computer, and the Adobe Premier software program (Adobe Systems Incorporated) was used to measure the time interval between each successive action step. The results are shown in Table 1 and reveal that, on average, the standard deviation of the ISI ranged between 20 and $35 \mathrm{msec}$ for the coherent events but between 220 and $375 \mathrm{msec}$ for the incoherent events. For the latter, the result of this higher variability was to render the sequence of action steps less rhythmical and temporally coordinated. Lastly, the four remaining scenes within the experiment were termed continuous because, in lieu of individual body movements within the event's total time span, the activity consisted primarily of one continuous movement. The total durations of coherent and incoherent events ranged between 9.1 and $11.6 \mathrm{sec}$, whereas those for continuous events were relatively shorter and ranged between 5.3 and $8.3 \mathrm{sec}$. Across all 12 scenes, there was an attempt to equate the degree of lighting, as well as the relative distance of the actors from the video camcorder. In all cases, no sound accompanied a given scene.

To ensure that the coherent, continuous, and incoherent events were perceived as varying in their degree of rhythmicity, a pretest was conducted with an independent group of 8 participants. They were instructed, while viewing each of the 12 scenes on a computer monitor, to tap during an event's duration (using a key on the computer console), so that their taps coincided with that point in time at which each individual nontemporal marker made contact with its 
Table 1

Set of Visual Scenes Used in Experiment 1, Categorized by Their Type of Event Structure, With the Total Duration of Each Scene and the Number, Duration, and Mean Interstimulus Interval (ISI) of the Various Action Steps

\begin{tabular}{lr}
\hline Event Structure $\quad$ Event Description \\
\hline Scene Set A
\end{tabular}

Coherent

Footsteps A male stands by a mailbox with a stack of letters in hand, walks up a driveway (16 strides with an ISI of $533 \pm 23 \mathrm{msec})$, and enters a garage door $(600 \mathrm{msec})$. Total duration $=9.1 \mathrm{sec}$

Typing A female, using one finger, types a short note on a typewriter (20 key strokes with an ISI of $545 \pm$ $20 \mathrm{msec})$ before hitting the return key $(200 \mathrm{msec})$. Total duration $=11 \mathrm{sec}$

Incoherent Bicycle

A male pedals down a street with the bike wobbling left to right (20 revolutions with an ISI of $550 \pm$ $120 \mathrm{msec}$ ) and then suddenly brakes and stops $(200 \mathrm{msec})$. Total duration $=11.2 \mathrm{sec}$.

Basketball A female dribbles a basketball with uneven bounce periodicities down the court (15 dribbles with an ISI of $600 \pm 250 \mathrm{msec}$ ) and makes a layup (300 msec). Total duration $=9.3 \mathrm{sec}$

Continuous

Frisbee

Hug

A female throws a Frisbee and at the end of its trajectory, it is caught by a male. Total duration $=5.3 \mathrm{sec}$. A male and female, facing one another, embrace and then release themselves. Total duration $=7.1 \mathrm{sec}$.

\section{Scene Set B}

Coherent Bicycle

Basketball

A male pedals down a street (21 revolutions with an ISI of $500 \pm 35 \mathrm{msec}$ ) and then suddenly brakes and stops $(200 \mathrm{msec})$. Total duration $=10.7 \mathrm{sec}$.

A female dribbles a basketball down the court (16 dribbles with an ISI of $550 \pm 30 \mathrm{msec}$ ) and makes a layup (300 msec). Total duration $=9.1 \mathrm{sec}$.

Incoherent Footsteps

A male stands by a mailbox with a stack of letters in hand, staggers up a driveway (15 steps with an ISI of $600 \pm 375 \mathrm{msec}$ ), and enters a garage door $(600 \mathrm{msec})$. Total duration $=9.5 \mathrm{sec}$.

Typing A female, using one finger, pecks a short note on a typewriter (19 key strokes with an ISI of $600 \pm$ $200 \mathrm{msec})$ before hitting the return key $(200 \mathrm{msec})$. Total duration $=11.6 \mathrm{sec}$.

Continuous Baseball

A male throws a baseball and it is caught by a female. Total duration $=5.4 \mathrm{sec}$

Drink A female picks up a glass of orange juice, drinks it, and sets the glass on a table. Total duration $=8.3 \mathrm{sec}$.

respective surface (i.e., a foot hitting the driveway, a finger hitting a typewriter key, a basketball hitting the floor, or a bicycle pedal being closest to the road). For the continuous events, the participants were told to coordinate their single tap for an event with the ending of the activity's trajectory (i.e., a Frisbee or baseball being caught, an end of an embrace, or a glass of orange juice being set on a table).

These data were then evaluated to determine the extent to which the tap periodicities that the participants produced deviated from each event's actual set of ISIs. An overall ANOVA confirmed that the degree of error in the synchronization responses varied as a function of event structure $[F(2,14)=12.09, p<.01]$. A set of Tukey post hoc comparisons showed that the magnitude of deviation was significantly higher for incoherent events $(M=38 \%)$ than for either coherent $(M=11 \%)$ or continuous $(M=9 \%)$ ones. These results therefore validate the main manipulation of the experiment by illustrating that the different categories of events described in Table 1 varied in their degree of temporal regularity.

The 12 scenes were then divided into two presentation sets (A and $\mathrm{B}$ ), each containing six scenes that varied in event structure (coherent, continuous, or incoherent). As can be seen in Table 1 , the scenes that were coherent in Set A were incoherent in Set B, and vice versa.

For the initial learning phase of the experiment, the six scenes within each set were organized into four blocks of randomized trials, to yield a total of 24 trials.

For the duration reproduction phase, the name of each of the six scenes within each set (A and B) was presented three times in a randomized format, for a total of 18 trials.

Apparatus. An RCA CPR250 video camcorder was used to film the original set of visual scenes, and these were then arranged into a given sequence for each presentation set (A and B) via a Panasonic AG-1950 editing system. During the initial learning phase of the experiment, the sequence of trials was presented on a JVC HR-D320 videocassette recorder interfaced with a 19-in. (48.3-cm) Toshiba C990 television. Duration judgments during the reproduction phase were collected on a set of Macintosh Performa 6115 CD computers.

Procedure. Forty-eight participants were randomly assigned to one of the two instructional groups (prospective or retrospective), and within each, 24 participants were randomly assigned to one of the two learning trial conditions (four or eight). Recorded instructions informed the participants of scene presentation details and task requirements. For the initial learning phase, the participants were told that the experiment involved a study of perceptual judgments and that they would be asked to rate a set of scenes on four different dimensions. In addition, the participants in the prospective conditions were instructed to attend to the total duration of each event because they would later be tested on this information in a second phase of the experiment. In the retrospective conditions, the participants were instructed to attend to and remember the sequence of actions within each event, and the upcoming duration reproduction task was not mentioned.

All the participants then received a set of learning trials in which a separate trial block was presented for each of the four rating tasks and the set of instructions for each task was administered before the block of six trials. On each trial, a 2-sec blackened scene served as a warning signal for a given activity that occurred $2 \mathrm{sec}$ later. During a 5-sec response period that followed, the participants were instructed to provide a rating judgment on a 10-point scale. For ratings of predictability, the participants were asked to decide whether the performance of the given activity seemed skilled and coordinated $(1=$ very predictable $)$ or relatively unskilled and uncoordinated. The second task of frequency ratings required the participants to judge whether the activity was one they saw on a regular basis $(1=$ very frequent $)$ or only occasionally. Interest ratings assessed a scene's ability to maintain a viewer's attention $(1=$ very interesting) or induce a sense of boredom. Lastly, for ease-of-remembering judgments the participants were asked to decide whether it would be relatively easy ( 1 = very easy) or difficult to later mentally recreate the scene from memory. All the participants performed these four rating tasks in the same order. The participants in the eight-trial learning condition were asked to immediately repeat this set of rating tasks a second time for "purposes of reliability."

Following this initial learning period, all the participants were then given a duration reproduction task. On each trial, a word was displayed on the computer screen (e.g., typing, Frisbee, or baseball) for $2 \mathrm{sec}$, which indicated which activity they would be asked to recall. Three seconds later, the word "start" appeared, and at this point, the participants were instructed to "mentally recreate the scene, from its beginning to end, in the way it exactly occurred over 
its original time course." The mental ending point of the scene was indicated by a handheld buttonpress that was automatically recorded by the computer. The participants were tested in small groups of 2-4 individuals, and the duration of an entire experimental session was approximately $35 \mathrm{~min}$ for the participants in the four-trial learning condition and $50 \mathrm{~min}$ for those in the eight-trial condition.

\section{Results}

Data from the rating and duration reproduction tasks were analyzed separately through a series of ANOVAs. Each will be discussed in turn.

Rating data. Table 2 depicts the mean ratings of predictability, frequency, interest, and ease of remembering as a function of the scenes' internal structures. Means have been collapsed over the number of learning trials, presentation set (A or B), and type of instructions administered to the participants, since these variables exerted negligible influences.

The set of predictability ratings validated the primary manipulation in this experiment. A main effect for event structure $[F(2,176)=17.4, p<.001]$ indicated that incoherent events were rated as less predictable $(M=7.17)$ than either coherent $(M=3.50)$ or continuous $(M=4.83)$ ones. A set of Tukey HSD tests confirmed that this difference was a significant one $(p<.01)$ and that the latter two conditions did not differ.

The three remaining sets of perceptual judgments yielded null effects. The frequency judgments produced an average of 3.83, indicating that all the visual scenes were highly familiar. The degree of interest was also comparable across all the visual scenes and produced ratings at the midpoint of the scale $(M=5.05)$. Lastly, the participants judged all three classes of event structures as equally easy to remember $(M=3.95)$, including the incoherent scenes that depicted unskilled actions.

Duration reproduction. Given that the experimental stimuli varied in their total durations, the data were standardized through a set of ratio scores for each participant that evaluated reproduced duration relative to a scene's actual duration (i.e., RD/AD). This particular measure has the advantage of parsimoniously expressing two types of information. First, it relates the directional bias of the participants' responses, in that ratios greater than or less than 1.00 indicate relative over- and underestimations, respectively. Second, the percentage of error in reproduced duration is expressed by subtracting each observed ratio from a value of 1 and then multiplying the absolute difference by 100 . For example, a ratio of 1.26 indicates that a scene

Table 2

Mean Ratings of Predictability, Frequency, Interest, and Ease of Remembering for Scenes in Experiment 1 as a Function of Event Structure

\begin{tabular}{lccc}
\hline & \multicolumn{3}{c}{ Event Structure } \\
\cline { 2 - 4 } Type of Perceptual Judgment & Coherent & Incoherent & Continuous \\
\hline Predictability & 3.50 & 7.17 & 4.83 \\
Frequency & 3.00 & 4.67 & 3.83 \\
Interest & 5.33 & 4.83 & 5.00 \\
Ease of remembering & 3.67 & 4.00 & 4.17 \\
\hline
\end{tabular}

was remembered as being $26 \%$ longer than its actual duration, and a ratio of . 74 as $26 \%$ shorter than its actual duration. These mean ratio values are depicted in Figure 1 as a function of event structure, number of learning trials, and type of duration judgment. An overall ANOVA for these three factors was conducted, and when significant differences emerged, they were further examined through a set of Tukey HSD tests in which $p$ was set to .05 .

The analysis revealed three major findings. First, there was a significant main effect for event structure $[F(2,176)=28.92, p<.001]$. Overall, incoherent durations were significantly less accurate than either coherent or continuous ones, which produced comparable degrees of error. In addition, all three event types tended to produce overestimations, but only those for incoherent scenes significantly differed from a value of 1.00 (representing no bias). On average, incoherent scenes were overestimated by $27 \%$, whereas coherent and continuous scenes were overestimated by $9 \%$. A secondary analysis revealed that this effect generalized to all instances within each of the two scene sets (A and B). Second, there was also a significant main effect for type of duration judgment $[F(1,176)=5.82, p<.05]$. Overall, judgments were significantly more accurate in the prospective condition than in the retrospective one.

Lastly, the overall ANOVA revealed that these effects were qualified by a significant three-way interaction between event structure, number of learning trials, and type of duration judgment $[F(2,176)=10.84, p<.001]$. First, consider performance in the prospective conditions shown in Figure 1, in which the participants knew in advance that a time judgment would be forthcoming. Consistent with the main effect of event structure, the durations of coherent and continuous scenes were more accurately reproduced than were those of incoherent scenes. In contrast to coherent and continuous scenes, which produced an equivalent level of performance that did not vary from a value of 1.00, incoherent scenes yielded significant overestimations. This overall pattern of results applied to both learning conditions, in that the latter variable exerted no effects on performance.

The retrospective conditions, on the other hand, yielded a somewhat different pattern of results. After four learning trials, the reproduced durations for all three event types were significantly less accurate than those in the prospective condition. In addition, although the durations of coherent and continuous scenes were more accurate than those of incoherent scenes, all event types yielded significant overestimations. However, with additional learning experience, the reproduced duration of coherent and continuous scenes significantly improved, so that estimates did not vary from a value of 1.00 and became as accurate as prospective ones. Incoherent durations, however, did not improve with learning and remained less accurate than prospective ones.

\section{Discussion}

The results of this first experiment demonstrate that duration estimates of visual events depend on at least 


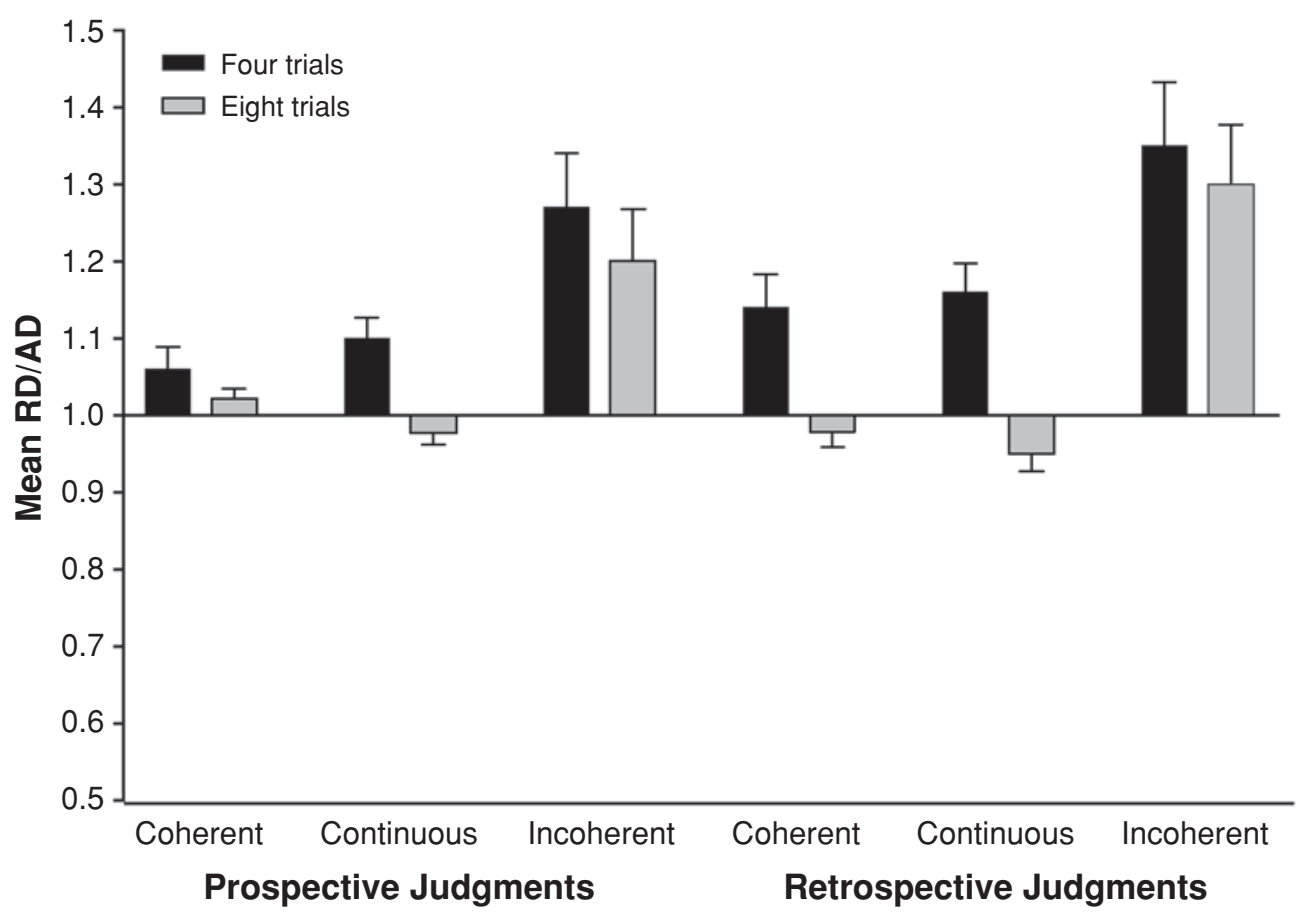

Figure 1. Mean ratio of participants' reproduced durations (RDs) to events' actual durations (ADs) in Experiment 1 as a function of event structure, number of learning trials, and type of duration judgment. Error bars represent the standard deviations of the participants' judgments.

three different factors - namely, type of duration judgment, event structure, and degree of learning experience. In accord with results in the past literature, prospective judgments yielded more accurate estimates than did retrospective ones (e.g., Brown, 1985; Miller, Hicks, \& Willette, 1978). This finding is typically attributed to different cognitive mechanisms that mediate experienced versus remembered duration (Zakay, 1989). In prospective situations, participants know in advance that a duration judgment will be forthcoming and can, therefore, engage in conscious timekeeping strategies (e.g., counting or tapping) to aid their performance. Retrospective judgments, on the other hand, are assumed to reflect a low level of accuracy, because attending is oriented primarily toward the event's nontemporal information and there is no reason to monitor time's passage. Although the present results revealed some support for these ideas, they also indicate that the accuracy of prospective and retrospective judgments depends on the inherent structure of an event, as well as on the relative degree of familiarity acquired through learning experience. This leads to situations in which retrospective judgments can be as accurate as prospective ones.

Across all the conditions in Experiment 1, coherent and continuous events consistently yielded more accurate estimates than did incoherent ones. This effect was a reliable one that generalized to both prospective and retrospective judgments and to both early and late stages of learning. It is also an effect that cannot be attributed to the individual instances varying in their overall frequency or interest level, factors that have been found to influence duration estimates. The perceptual rating data demonstrated that all three classes of events were judged comparably on these dimensions. In addition, the same scenes were used in both the coherent and the incoherent conditions of the experiment and were relatively matched in total duration, thus reducing any experimental error that might arise from differential event characteristics.

Within the present framework, the effect of event structure on time estimation behavior is assumed to stem from the relative relationship between an event's temporal and nontemporal information and the resulting consequences for encoding and remembering abilities. In coherent events, the set of nontemporal markers predictably recurs with an invariant temporal periodicity, which gives rise to a characteristic rhythm, rate, and total duration. Continuous events display a similar structure, except that there are only two nontemporal markers (i.e., the beginning and end) that enclose one temporal periodicity. In either case, the two dimensions of temporal and nontemporal information are inextricably entwined and serve to jointly define the event itself. With sufficient learning experience, this structural array allows both dimensions to be jointly encoded into the cognitive system, so that attending to one leads to an incidental learning of the other.

In the prospective condition of Experiment 1, this encoding process was facilitated by the structural predictability of event information. Given that the participants knew in advance that a duration judgment would be required, they could easily track (or count or tap to) the array 
of nontemporal markers that unfolded with an invariant periodicity and then use these as an effective retrieval scheme to recapitulate the event's duration. As the present data indicate, a high degree of accuracy emerged even with minimal learning experience: Performance after four learning trials was comparable to that after eight trials and did not vary from a ratio of 1.00 , representing perfect accuracy. The more interesting results, however, lie in the retrospective conditions of the experiment. Although traditional models of time estimation behavior have assumed that duration is not reliably encoded in retrospective situations (e.g., Zakay, 1989), coherent and continuous events displayed a mean absolute error value of 13\% after four learning trials and, with additional learning, only $2 \%$ mean absolute error. The latter value, in fact, did not significantly vary from a ratio of 1.00 and was as accurate as performance in the prospective condition. These results, then, support the idea that with sufficient opportunity to discern the inherent structural relationship between temporal and nontemporal information, both can be jointly encoded into the cognitive system, so that attending to one dimension leads to an incidental learning of the other.

Incoherent events, on the other hand, led to a very different pattern of results. Duration estimates not only were highly inaccurate, but also did not improve with additional learning experience. These types of events are characterized by an array of nontemporal markers that recur with a more variable temporal periodicity. Hence, the arrays of temporal and nontemporal information seem structurally dissociated from one another, in that there is no lawful rhythm according to which nontemporal items appear. As the present results suggest, this in turn leads to an independent encoding strategy. Although duration reproduction was relatively more accurate in prospective situations, in which the participants were able to engage in conscious timekeeping strategies, performance declined significantly in retrospective situations, in which attending was directed toward the array of nontemporal information. Nonetheless, even in the former condition, the mean absolute error rate was quite high at $23 \%$. Given that the sequence of nontemporal information (i.e., body movements) unfolds with an irregular periodicity, it is more difficult to track (or count or tap to) their recurrence over the event's total time span. In effect, there are no structural referents that can reliably be used for either attending or retrieval.

In sum, then, these overall findings directly converge with those in previous research in which the remembered duration of auditory events was examined (Boltz, 1992, 1998, 1999) and suggest that effects of event structure and learning experience across different judgment paradigms generalize to events from different modalities. This finding serves to qualify the work of Glenberg and Jona (1991), who found that people not only are better able to reproduce rhythms in the auditory versus the visual modality, but also do not benefit from the effects of more predictable rhythms when these are visually presented. The present set of results suggests that these findings do not apply to duration judg- ment tasks. Even though rhythm may be easier to remember when it occurs in an auditory format, it appears to exert a common impact upon the ability to learn and remember event durations from different modalities.

The final finding of interest is the directional bias of estimates that appeared in some conditions of the experiment. Although the duration estimates for coherent and continuous events were bias free (i.e., did not vary from a ratio of 1.0) in both learning groups in the prospective condition, as well as in the eight-trial group in the retrospective condition, significant overestimations appeared when the participants retrospectively judged these event durations after four learning trials. This overestimation bias was even more pronounced for incoherent events in all the conditions in the experiment. This effect has been observed in past studies that have manipulated both learning and event structure (e.g., Boltz, 1995, 1999; Boltz et al., 1998) and has been suggested to stem from an availability heuristic (a la Block, 1985; Ornstein, 1969). In those situations in which event duration is not reliably encoded into the cognitive system, people appear to base their judgments on the most salient information available in memory - namely, the number of unrelated chunks or changes within the nontemporal array. Incoherent durations will seem particularly long, due to the overall lack of temporal coordination that renders a set of individual movements that appear temporally dissociated and unrelated to one another. Similarly, the high degree of predictability within coherent and continuous events may not be appreciated as such at early stages of learning. Because the invariant periodicity of nontemporal markers is not yet fully apparent, people are biased by the relative number of action segments in memory. The inherent predictability of the event renders a fewer number of such segments than in incoherent events, but the result in both cases is reduced accuracy in the form of an overestimation bias.

\section{EXPERIMENT 2}

Although Experiment 1 demonstrated that people can incidentally learn and remember visual durations in much the same way as they do auditory ones, several additional issues merit further investigation. The first involves a direct comparison of auditory and visual events, to determine whether one modality produces better learning and remembered duration performance than does the other. One possibility is that the two modalities will produce comparable levels of accuracy. The error rate in judging the visual durations of Experiment 1 approximated that previously reported for auditory durations (Boltz, 1992, 1995, 1999), and given that both modalities are influenced by event structure and learning, the same underlying cognitive mechanisms may be at play. On the other hand, evidence for the temporal distinctiveness hypothesis (Glenberg \& Swanson, 1986) may be manifested as a superiority effect for the auditory modality. Auditory rhythms are more easily learned (Handel \& Buffardi, 1968) and better remembered (Glenberg \& Jona, 1991) 
than visual ones, and if this effect applies to the present context, people may reveal an enhanced ability to remember auditory durations after fewer learning trials than is true for the visual modality.

A related issue concerns how duration judgments vary between situations in which people experience one modality alone rather than the two together. No differences should emerge if the information provided by audition and vision is redundant. However, if duration accuracy increases with combined modality information, this would suggest that auditory events (or visual ones) afford certain cognitive skills that the other modality does not. For example, it may be the case that auditory and visual imagery together are more effective for retrieval and remembering processes than is the use of one type of imagery alone.

In addition to investigating the relative accuracy of events, Experiment 2 also examined whether there would be a differential pattern of bias as a function of modality. The past literature has frequently reported that whereas visual (V) events are judged to be shorter than auditory (A) ones, auditory and audiovisual (AV) events produce comparable levels of performance (i.e., $\mathrm{V}<\mathrm{A}=\mathrm{AV}$; see, e.g., Goldstone \& Lhamon, 1972, 1974; Walker \& Scott, 1981). To date, it is not clear why this bias occurs and whether it represents over- and underestimations, respectively, relative to events' actual durations, or simply two different magnitudes of over- or underestimation. However, one can address this issue by evaluating the ratio of reproduced duration relative to actual duration. This, in turn, will provide insight into whether the underlying mediational mechanisms are the same in auditory and visual events or whether each is subject to its own set of encoding and retrieval processes.

These various issues were investigated in a design very similar to that in Experiment 1. During an initial encoding phase, different groups of participants experienced six events in their auditory, visual, or audiovisual modality for a varying number of learning trials. Half of the participants were informed in advance of the upcoming time estimation task (i.e., prospective), whereas the remainder were not (i.e., retrospective). Afterward, each participant was asked to reproduce the total duration of each event through a buttonpress. In contrast to Experiment 1, all the events were coherent in their internal structure and contained a predictably recurrent series of items, so that performance could be observed at its optimal level.

\section{Method}

Design and Participants. The design was a $3 \times 2 \times 2$ betweensubjects factorial. Three groups of participants were presented with the same set of events in the auditory, visual, or audiovisual modality. Within each of these conditions, the number of exposures to each event in the initial learning phase (four or eight), as well as the type of duration judgment (prospective or retrospective), was also manipulated.

One hundred ninety-two students from an introductory psychology class at Haverford College participated in the experiment for course credit. Sixteen were randomly assigned to each of the 12 experimental conditions.
Table 3

Set of Stimuli Used in Experiment 2, With the Total Duration of Each Event and the Number, Duration, and Mean Interstimulus Interval (ISI) of the Various Action Steps

\begin{tabular}{|c|c|}
\hline Event & Description \\
\hline Footsteps & $\begin{array}{l}\text { A male stands by a mailbox with a stack of letters in hand, } \\
\text { walks up a concrete driveway ( } 16 \text { steps with an ISI of } 533 \\
\pm 23 \mathrm{msec}) \text {, and opens a creaky garage door }(600 \mathrm{msec}) \text {. } \\
\text { Total duration }=9.1 \mathrm{sec} \text {. }\end{array}$ \\
\hline Basketball & $\begin{array}{l}\text { A female dribbles a basketball across the length of a court } \\
\text { (16 dribbles with an ISI of } 550 \pm 30 \mathrm{msec} \text { ) and makes a } \\
\text { layup ( } 300 \mathrm{msec}) \text {. Total duration }=9.1 \mathrm{sec} \text {. }\end{array}$ \\
\hline Clapping & $\begin{array}{l}\text { A female, watching a football game on TV, begins clapping } \\
\text { ( } 12 \text { claps with an ISI of } 800 \pm 25 \mathrm{msec}) \text { and then whistles } \\
\text { in victory }(900 \mathrm{msec}) . \text { Total duration }=10.5 \mathrm{sec} \text {. }\end{array}$ \\
\hline Knocking & $\begin{array}{l}\text { A male, standing in front of a door to a house, begins } \\
\text { knocking (nine knocks with an ISI of } 750 \pm 20 \text { msec) } \\
\text { and then opens a creaking door ( } 700 \mathrm{msec} \text { ). Total dura- } \\
\text { tion }=7.4 \text { sec. }\end{array}$ \\
\hline Sawing & $\begin{array}{l}\text { A male uses a handsaw to saw a board ( } 13 \text { strokes with an } \\
\text { ISI of } 750 \pm 15 \mathrm{msec}) \text {, and the board's end hits a concrete } \\
\text { floor }(300 \mathrm{msec}) \text {. Total duration }=10.05 \mathrm{sec} \text {. }\end{array}$ \\
\hline Eating & $\begin{array}{l}\text { A female takes a large bite from an apple }(500 \mathrm{msec}) \text {, } \\
\text { chews it }(17 \text { chews with an ISI of } 525 \pm 15 \mathrm{msec}) \text {, } \\
\text { and then throws the remainder into a metal trashcan } \\
(400 \mathrm{msec}) \text {. Total duration }=9.7 \mathrm{sec} \text {. }\end{array}$ \\
\hline
\end{tabular}

Stimulus materials. Six different visual scenes, enacted by the same male or female actor as in Experiment 1, were filmed onto videotape and are described in Table 3. All the events were coherent in their internal structure, in that a rhythmical and regularly recurrent set of body movements occurred within a total time span marked by a nonarbitrary beginning and end. The ISIs for movements within a given event were measured using the same procedure as that in Experiment 1, and as is indicated in Table 3, these displayed a relatively low degree of variability that ranged from 15 to $30 \mathrm{msec}$. In each case, an auditory sound coincided with a visual movement to create both an auditory and a visual rhythm within each event. The total time span of these events ranged between 7.4 and $10.5 \mathrm{sec}$.

To determine the psychological validity of the events' rhythmic structure, a pretest was conducted in which 24 independent participants were assigned to one of the three modality conditions. As in Experiment 1, they were asked to tap in synchrony with the auditory, visual, or audiovisual point of contact of each nontemporal marker with its respective surface. When the deviations of these taps were compared within an event's actual sequence of ISIs, the results of an overall ANOVA indicated no significant effects of either event instance or modality. The average degree of deviation was $9 \%$ across the entire experiment. These results, in conjunction with those from the computer analysis, thereby confirm that the set of events used in this experiment displayed a relatively invariant rhythm on both a structural and a perceptual/motor level.

For the initial learning phase of the experiment, the six events were arranged into four randomized blocks of trials, to yield a total of 24 trials. Three different versions of this set of learning trials were created. Although each displayed the same randomized ordering of events, the modality of event presentation varied. One version consisted of a videotape in which the set of scenes, along with their accompanying audio information, was displayed. In the second version, the visual scenes alone were presented in the absence of the soundtrack, whereas in the third, the audio information was presented alone in the presence of a blackened screen. Across these three sets, the total duration of a given event was equivalent and corresponded to the values in Table 3 . 
For the duration reproduction task, each of the names of the six events was presented three times in a randomized format for a total of 18 trials. Two such trial blocks (I and II) were created that simply varied in the ordering of trial presentation.

All other aspects of the method and procedure were identical to those in Experiment 1.

\section{Results}

Results from the perceptual rating and duration reproduction tasks were once again analyzed separately through a series of ANOVAs, and each will be discussed in turn.

Rating data. Each of the four perceptual ratings yielded null effects. The ease-of-remembering, degree-of-interest, and frequency ratings did not vary across the different experimental conditions and produced overall means of $3.19,5.21$, and 4.72 , respectively. The predictability ratings also revealed that all six events within the experiment were judged to have comparable levels of high internal coherence $(M=3.68)$.

Duration reproduction. As in Experiment 1, both the accuracy and the directional bias of the duration reproduction data were assessed through a set of ratio scores (i.e., $\mathrm{RD} / \mathrm{AD})$. The overall mean performance in the different experimental groups is depicted in Figure 2. These means have been collapsed over the two different counterbalance orders, since this variable exerted no significant effects upon behavior.

The most important finding is that there were no significant effects due to event modality: The visual, auditory, and audiovisual presentations of events yielded similar mean ratio scores of $1.03,1.02$, and 1.02, respectively. In addition, although there was a significant main effect of trials $[F(1,180)=4.67, p<.05]$, in which eight exposures to a given event yielded greater accuracy $(M=4 \%$ error) than did four exposures ( $M=9 \%$ error), the magnitude of improvement in duration recall was comparable among the three modalities. Figure 2 also suggests there is an overestimation bias for all events after four learning trials but an underestimation bias after eight learning trials. A set of Tukey HSD tests revealed that whereas the degree of overestimation was significant $(p<.05)$, the magnitude of underestimation was not.

Lastly, a significant interaction between the number of learning trials and judgment type $[F(1,180)=5.23$, $p<.05]$ revealed that prospective judgments were more accurate $(M=4 \%$ error $)$ than retrospective ones $(M=$ $14 \%$ error) after four exposures $(p<.05)$ but displayed comparable levels of accuracy after eight exposures $(M=$ $3 \%$ vs. $5 \%$, respectively). Again, this pattern of results applied to all three modalities.

\section{Discussion}

The results from this second experiment reveal that duration estimates are independent of their particular modality. Regardless of whether participants encountered events in their auditory, visual, or audiovisual format, all were remembered with comparable levels of accuracy, relative to their actual durations. This effect applied to both prospective and retrospective judgments. The ability

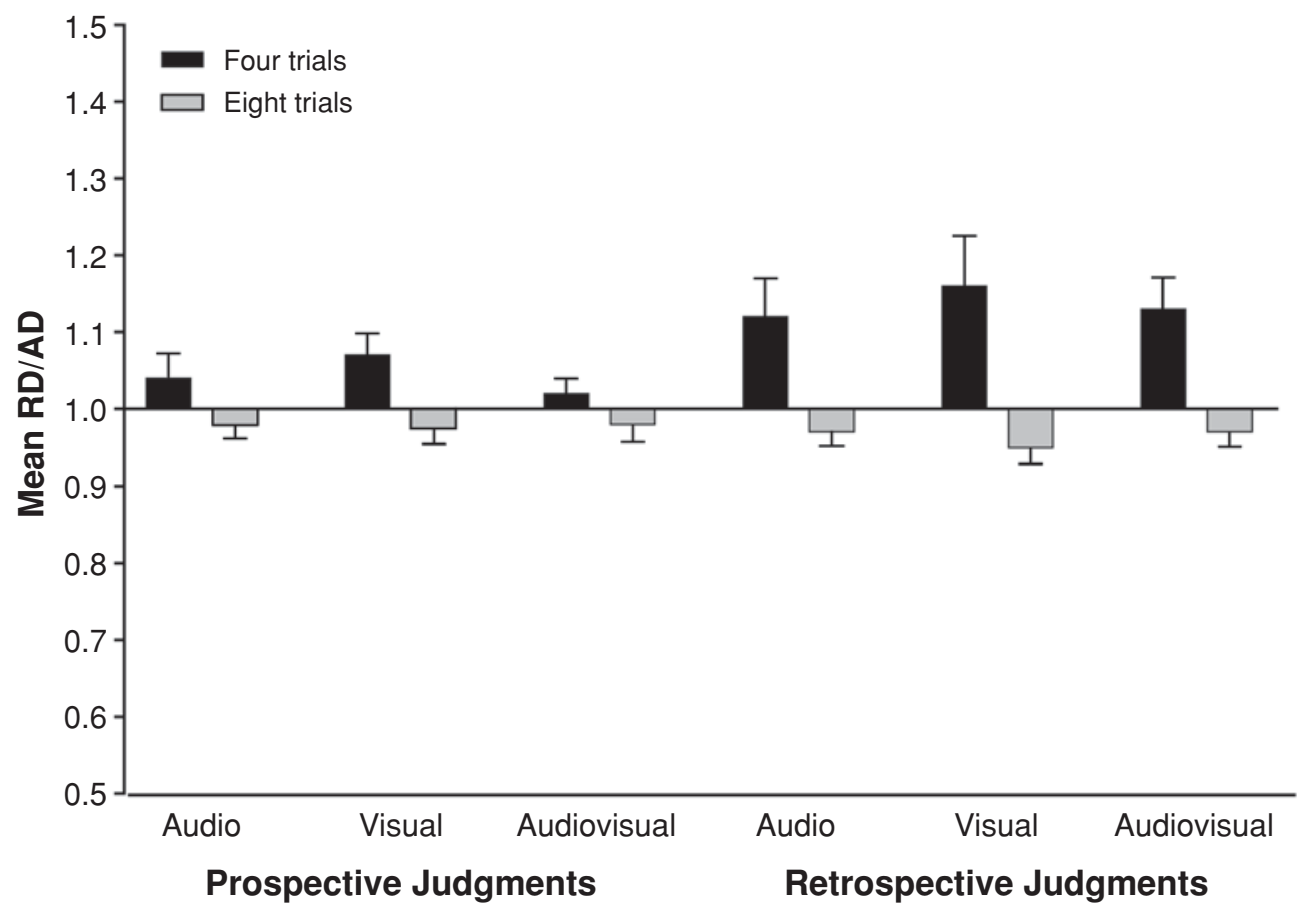

Figure 2. Mean ratio of participants' reproduced durations (RDs) to events' actual durations (ADs) in Experiment 2 as a function of event modality, number of learning trials, and type of duration judgment. Error bars represent the standard deviations of the participants' judgments. 
to consciously monitor time facilitated performance at early stages of learning in which the inherent organization of events was not yet fully apparent, but this was true for events in all modalities. In addition, no modality effects were observed at different stages of learning. Although the accuracy of estimates was significantly greater after eight than after four trials of learning, the magnitude of this improvement was equal across the three modality conditions. The finding is interesting because previous research has shown that auditory rhythms are acquired and more easily reproduced than visual ones after fewer learning trials (Handel \& Buffardi, 1968), leading one to expect an auditory advantage for remembered duration at earlier stages of learning. Given that this effect did not emerge, even with the simplest type of rhythm (i.e., isochronous), this further supports the idea that the same underlying cognitive mechanisms mediate duration judgments, regardless of event modality.

The second major finding from this experiment was the lack of bias effects as a function of event modality. Several studies in the literature have reported that auditory and audiovisual events are judged to be significantly longer than visual ones when duration estimates are observed in prospective situations (Goldstone \& Goldfarb, 1964; Goldstone \& Lhamon, 1972, 1974; Walker \& Scott, 1981; Wearden et al., 1998). Although it is unclear whether the overestimation bias for auditory events is relative to the events' actual durations or merely relative to the durations of visual events, it did not emerge here for either prospective or retrospective judgments. Instead, the only type of directional error to appear was an overestimation bias of events from all modalities after four trials of learning. This finding converges with the results of Experiment 1 as well as with those of earlier studies (Boltz, 1999; Boltz et al., 1998). At early stages of learning, the inherent predictability of event structure is not yet fully apparent, and so people are biased by the relative number of action segments.

\section{GENERAL DISCUSSION}

The results from the two experiments reported here suggest that the same underlying cognitive mechanisms are responsible for mediating event durations from different modalities. Experiment 1 showed that the effects of learning and event structure that had previously been observed for auditory events also apply to visual ones. In both cases, the durations of predictably structured events are remembered better than those of incoherent ones, and this applies to both prospective and retrospective judgments. Prospective paradigms afford greater duration judgment accuracy by encouraging the use of conscious timekeeping strategies (e.g., tapping or counting) to perform the task at hand. Relative to retrospective judgments, prospective ones were, in fact, more accurate for both coherent and incoherent events, and this was particularly true for the former, whose internal structure ensures a greater ease of time monitoring. However, after more ex- tensive learning experience in which the events' intrinsic organization became more apparent, retrospective judgments of coherent events were as accurate as prospective ones. Only judgments of incoherent durations remained inaccurate in retrospective situations, regardless of the amount of learning. This grants further support to the idea that the joint relationship between the temporal and the nontemporal structures of coherent events allows both to be encoded in a unitary fashion, thereby allowing duration to be incidentally learned even when attention is directed toward nontemporal information. Incoherent events, on the other hand, display an array in which temporal and nontemporal information are structurally dissociated from one another. In order for encoding to occur, each dimension must be attended to independently of the other, so that duration is relatively well remembered only in prospective situations.

These differences in cognitive processing activities as a function of event structure apply to both auditory and visual events. Experiment 2 further revealed that when duration memory is directly compared between coherent events from the auditory, visual, and audiovisual modalities, no differences emerge. Although the previous literature has yielded mixed results, the null effects observed here are inconsistent with the results in other studies that have shown that auditory events produce greater discrimination accuracy (Grondin et al., 1998; Grondin \& Metthé, 1993; Grondin \& Rousseau, 1991) and longer estimates (Goldstone \& Goldfarb, 1964; Goldstone \& Lhamon, 1972, 1974; Walker \& Scott, 1981; Wearden et al., 1998) than do visual events. More generally, the lack of modality effects diverges from the temporal distinctiveness theory (Glenberg \& Swanson, 1986), in which the auditory modality is deemed to have greater temporal discrimination skills than does the visual one.

This discrepancy between the present and the past research may, in part, be due to methodological differences. Studies reporting modality differences have typically relied on much shorter durations (i.e., less than $1 \mathrm{sec}$ ) than those used here (ranging between 5.3 and $11.6 \mathrm{sec}$ ), which may invoke different processing mechanisms. Second, the prospective paradigm used in past research has been oriented toward the study of experienced duration, and consequently, participants have traditionally been asked to make duration estimates immediately after an event's presentation. In contrast, the present research relied on a different methodological strategy. Although the participants in the prospective versus retrospective conditions varied in their preknowledge of the upcoming time estimation task, both performed the duration reproduction task after a time delay — namely, the initial learning phase. Given that the same procedure was used for both groups, this allowed one to directly compare their performances. Nonetheless, remembered duration was assessed in each case. Hence, it may be the case that modality differences emerge only during experienced duration, which is what the previous research has exclusively investigated. 
A third issue has been highlighted by the work of Penney et al. (1998), who noted that modality effects in time estimation behavior are more likely to emerge in within-subjects designs. According to these authors, the internal clock runs at a faster rate for auditory than for visual stimuli, which means that the accumulated clock value for a given duration will be greater for auditory events. Since this contrast effect is more perceptually salient when the two modalities are directly compared, this results in auditory events appearing to be longer than visual ones. Experiment 2, in contrast, relied on a between-subjects design, which Penney et al. argued is less likely to yield modality differences. Although this may be true, a between-subjects design was necessary for the present research. One goal of the present experiments was to examine duration judgments in prospective versus retrospective situations, and it is not immediately clear how different modalities can be directly compared with one another in a retrospective paradigm.

A between-subjects design can also be argued to be more representative of everyday contexts. We rarely compare the auditory and visual modes of the same event, although there may be situations in which such a comparison occurs. More typically, when both modalities are not simultaneously available, our spatial location is such that we can hear an event but not see it, or alternatively, our distance from a scene enables us to see but not hear the event transpiring. In other words, our selective attention is focused primarily on one modality or the other. Hence, it is worthwhile to ask whether one's attentional focus results in different judged duration abilities, and the research here suggests that it does not.

This highlights another factor that may help to explain the discrepancy between the past and the present research: the use of different types of experimental stimuli. An auditory advantage for rhythmic information may be more likely to emerge with very simplistic events, such as monotonic or flashing light sequences, in which each individual nontemporal item is indistinguishable from the others. If the auditory modality does, in fact, have better temporal processing skills than does the visual one, this ability may be more apparent in situations in which the sequence of nontemporal information is relatively uniform and lacks distinctiveness. However, within the context of everyday activities, this advantage may disappear, due to an inherent structural organization of visual events that is directly correlated with a lawful sequence of sound. Many everyday activities are schematically governed, in which individual actions occur in a prescribed temporal ordering and unfold toward a given goal (Bower, Black, \& Turner, 1979). In addition, Newtson (1973) has described how such activities are organized into lower level action units whose boundaries are perceptually salient. In many cases, the boundaries of these action units are accompanied by a sound source (e.g., the sound of footsteps, the dribbling of a basketball, or the keystrokes of a typist), which serves to reinforce the organization of visual information and, thereby, to provide a joint array of predictably recurrent markers within the event's total time span. Within the pre- test of both Experiments 1 and 2, it was found that people can reliably synchronize to these markers, with low variability. This structural array is also suggested to aid remembering by providing an effective retrieval scheme in which to reimagine an event's total time span, from beginning to end, and regardless of whether only one modality is available for perception. Given that audio information and visual information are structurally correlated and are redundant, each affords an efficient recapitulation of an event's total duration. And as was found here, people are able to do this quite accurately - especially when the event's internal structure is a coherent and predictable one and a sufficient amount of learning experience has occurred. In sum, then, duration judgments of naturalistic events may involve a different set of cognitive mechanisms than do those arising from more traditional laboratory stimuli. This, in turn, demands a more careful analysis of an event's internal structure and its relative degree of familiarity, in order to determine the nature of the encoding process and whether input of the event's temporal and nontemporal information into the cognitive system is apt to occur jointly or independently.

The idea that duration information from the auditory and visual modalities is processed by a centralized mechanism is not a new one and has been suggested by others in the previous literature (e.g., Bobko et al., 1977; Creelman, 1962; Treisman, 1963). It is worthwhile to briefly consider whether this is adaptive from a cognitive evolutionary perspective. One can argue that, in fact, it is. If a common mechanism is used for the judged duration of a wide range of environmental events, regardless of their particular modality, this provides a parsimonious system in which the same processing activities are at play. This, in turn, provides a system that is less vulnerable to interference effects. Many naturalistic events are multimodal, and using the same mechanisms to process this audiovisual array would ensure a more reliable representation of an event's total duration. Lastly, on a behavioral level, a centralized processor for modality information increases the overall probability of remembering in different contexts. Individuals do not always have access to both modalities at once (e.g., due to medical infirmities or their location and distance from a source), but if the audio and the visual information for a given event are structurally correlated with one another, this will ensure that duration will be remembered with the same level of accuracy when one modality is available, rather than both.

\section{REFERENCES}

BERry, W. (1976). Structural functions in music. Englewood Cliffs, NJ: Prentice Hall.

Bertelson, P. (1999). Ventriloquism: A case of crossmodal perceptual grouping. In G. Aschersleben, T. Bachmann, \& J. Müsseler (Eds.), Cognitive contributions to the perception of spatial and temporal events (pp. 347-362). Amsterdam: Elsevier.

Block, R. A. (1985). Contextual coding in memory: Studies of remembered duration. In J. A. Michon \& J. L. Jackson (Eds.), Time, mind, and behavior (pp. 169-178). Heidelberg: Springer.

Boвко, D. J., Thompson, J. G., \& Schiffman, H. R. (1977). The per- 
ception of brief temporal intervals: Power functions for auditory and visual stimulus intervals. Perception, 6, 703-709.

BoLtZ, M. G. (1991). Some structural determinants of melody recall. Memory \& Cognition, 19, 239-251.

Boltz, M. G. (1992). The remembering of auditory event durations. Journal of Experimental Psychology: Learning, Memory, \& Cognition, 18, 938-956.

Boltz, M. G. (1994). Changes in internal tempo and effects on the learning and remembering of event durations. Journal of Experimental Psychology: Learning, Memory, \& Cognition, 20, 1154-1171.

Boltz, M. G. (1995). Effects of event structure on retrospective duration judgments. Perception \& Psychophysics, 57, 1080-1096.

BoLTZ, M. G. (1998). The processing of temporal and nontemporal information in the remembering of event durations and musical structure. Journal of Experimental Psychology: Human Perception \& Performance, 24, 1087-1104.

Boltz, M. G. (1999). The processing of melodic and temporal information: Independent or unified dimensions? Journal of New Music Research, 28, 67-79.

Boltz, M. G., Kupperman, C., \& Dunne, J. (1998). The role of learning in remembered duration. Memory \& Cognition, 26, 903-921.

Bower, G. H., BLACK, J., \& TuRner, T. (1979). Scripts in memory for text. Cognitive Psychology, 11, 177-220.

Brown, S. W. (1985). Time perception and attention: The effects of prospective versus retrospective paradigms and task demands on perceived duration. Perception \& Psychophysics, 38, 115-124.

Brown, S. W., \& Stubbs, D. A. (1992). Attention and interference in prospective and retrospective timing. Perception, 21, 545-557.

Creelman, C. D. (1962). Human discrimination of auditory duration. Journal of the Acoustical Society of America, 34, 582-593.

Crowder, R. G., \& Green, R. L. (1987). On the remembrance of times past: The irregular list technique. Journal of Experimental Psychology: General, 116, 265-278

ForTIN, C. (1999). Short-term memory in time interval production. International Journal of Psychology, 34, 308-316.

Gebhard, J. W., \& Mowbray, G. H. (1959). On discriminating the rate of visual flicker and auditory flutter. American Journal of Psychology, 72, 521-529.

GlenberG, A. M., \& Jona, M. (1991). Temporal coding in rhythm tasks revealed by modality effects. Memory \& Cognition, 19, 514-522.

Glenberg, A. M., Mann, S., Altman, L., Forman, T., \& Procise, S. (1989). Modality effects in the coding and reproduction of rhythms. Memory \& Cognition, 17, 373-383.

Glenberg, A. M., \& Swanson, N. G. (1986). A temporal distinctiveness theory of recency and modality effects. Journal of Experimental Psychology: Learning, Memory, \& Cognition, 12, 3-15.

Goldman-EIsLER, F. (1972). Pauses, clauses, sentences. Language \& Speech, 15, 103-113.

Goldstone, S., \& Goldfarb, J. L. (1964). Direct comparison of auditory and visual durations. Journal of Experimental Psychology, 67, $483-485$

Goldstone, S., \& Lhamon, W. T. (1972). Auditory-visual differences in human temporal judgment. Perceptual \& Motor Skills, 34, 623633.

Goldstone, S., \& Lhamon, W. T. (1974). Studies of auditory-visua differences in human time judgment: I. Sounds are judged longer than lights. Perceptual \& Motor Skills, 39, 63-82.

Grondin, S. (2001). From physical time to the first and second moments of psychological time. Psychological Bulletin, 127, 22-44.

Grondin, S., Meilleur-Wells, G., Ouellette, C., \& Macar, F. (1998). Sensory effects of judgments of short-time intervals. Psychological Research, 61, 261-268.

Grondin, S., \& MetThé, L. (1993). Procedural effects on duration discrimination. In A. Garriga-Trillo, P. Minon, C. Garcia-Gallego, P. Lubin, J. Merino, \& A. Villarino (Eds.), Fechner Day 93: Proceedings of the Ninth Annual Meeting of the International Society for Psychophysics (pp. 107-112). Palma de Mallorca, Spain: International Society for Psychophysics.

Grondin, S., \& Rousseau, R. (1991). Judging the relative duration of multimodal short empty time intervals. Perception \& Psychophysics, $49,245-256$
Grosjean, F. H., Grosjean, L., \& Lane, H. (1979). The patterns of silence: Performance structures in sentence production. Cognitive Psychology, 11, 58-81.

HANDEL, S. (1988). Space is to time as vision is to audition: Seductive but misleading. Journal of Experimental Psychology: Human Perception \& Performance, 14, 315-317.

Handel, S., \& Buffardi, L. (1968). Pattern perception: Integrating information presented in two modalities. Science, 162, 1026-1028.

Holleran, S., \& Jones, M. R. (2001, November). Structure and timing of auditory and visual sequences. Paper presented at the 42nd Annual Meeting of the Psychonomic Society, Orlando, FL.

Jones, M. R., \& Boltz, M. G. (1989). Dynamic attending and responses to time. Psychological Review, 96, 459-491.

KowAL, K. H. (1976). Apparent duration of long meaningful events and meaningless intervals. Memory \& Cognition, 4, 215-220.

Kowal, K. H. (1981). Growth of apparent duration: Effect of melodic and non-melodic tonal variation. Perceptual \& Motor Skills, 52, 803817

Kubovy, M. (1988). Should we resist the seductiveness of the space: time::vision:audition analogy? Journal of Experimental Psychology: Human Perception \& Performance, 14, 318-320.

Large, E. W., \& Jones, M. R. (1999). The dynamics of attending: How people track time-varying events. Psychological Review, 106, 119159

LERDAHL, F., \& JACKENDOFF, R. (1983). A generative theory of tonal music. Cambridge, MA: MIT Press.

MaCAR, F., Grondin, S., \& CASINI, L. (1994). Controlled attention sharing influences time estimation. Memory \& Cognition, 22, 673-686.

MarTin, J. G. (1972). Rhythmic (hierarchical) versus serial structure in speech and other behavior. Psychological Review, 79, 487-509.

Metcalfe, J., Glavanov, D., \& Murdock, M. (1981). Spatial and temporal processing in the auditory and visual modalities. Memory \& Cognition, 9, 351-359.

Miller, G. W., Hicks, R. E., \& Willette, M. (1978). Effects of concurrent verbal rehearsal and temporal set upon judgments of temporal duration. Acta Psychologica, 42, 173-179.

MoHan, V. (1975). The influence of stimulus-modality, duration, and knowledge of results on improvement in temporal estimations. Manas, 22, 159-167.

Newtson, D. (1973). Attribution and the unit of perception of ongoing behavior. Journal of Personality \& Social Psychology, 28, 28-38.

Ornstein, R. (1969). On the experience of time. Baltimore: Penguin.

Penney, T. B., Allan, L. G., Meck, W. H., \& Gibbon, J. (1998). Memory mixing in duration bisection. In D. A. Rosenbaum \& C. E. Collyer (Eds.), Timing of behavior: Neural, psychological, and computational perspectives (pp. 165-193). Cambridge, MA: MIT Press.

Pfordresher, P. Q. (2003). The role of melodic and rhythmic accents in musical structure. Music Perception, 20, 431-464.

Piston, W. (1978). Harmony (4th ed., rev. and expanded by M. DeVoto). New York: Norton.

Posner, M. I., Nissen, M. J., \& Klein, R. M. (1976). Visual dominance: An information-processing account of its origins and significance. Psychological Review, 83, 157-171.

SHIPLEY, T. (1964). Auditory flutter-driving of visual flicker. Science, 145, 1328-1330.

Treisman, M. (1963). Temporal discrimination and the indifference interval: Implications for a model of the "internal clock." Psychological Monographs, 77, 1-31.

VON STURMER, G. (1966). Stimulus variation and sequential judgements of duration. Quarterly Journal of Experimental Psychology, 18, 354-357.

WALKER, J. T., \& SCOTT, K. J. (1981). Auditory-visual conflicts in the perceived duration of lights, tones, and gaps. Journal of Experimental Psychology: Human Perception \& Performance, 7, 1327-1339.

Warm, J. S., Greenberg, L. F., \& Dube, C. S. (1969). Stimulus and motivational determinants of temporal perception. Journal of Psychology: Interdisciplinary \& Applied, 58, 243-248.

Wearden, J. H., Edwards, H., Fakhri, M., \& Percival, A. (1998). Why "sounds are judged longer than lights": Applications of a model of the internal clock in humans. Quarterly Journal of Experimental Psychology, 51B, 97-120.

WelCH, R. B. (1999). Meaning, attention, and the "unity assumption" in 
the intersensory bias of spatial and temporal perception. In G. Aschersleben, T. Bachmann, \& J. Müsseler (Eds.), Cognitive contributions to the perception of spatial and temporal events (pp. 371-387). Amsterdam: Elsevier.

WeLCH, R. B., \& WARREN, D. H. (1980). Immediate perceptual response to intersensory discrepancy. Psychological Bulletin, 88, 638-667.

Witherspoon, D., \& Allan, L. G. (1985). The effect of a prior presentation on temporal judgments in a perceptual identification task. Memory \& Cognition, 13, 101-111.
ZAKAY, D. (1989). Subjective time and attentional resource allocation: An integrated model of time estimation. In I. Levin \& D. Zakay (Eds.), Time and human cognition (pp. 365-397). Amsterdam: Elsevier.

(Manuscript received March 1, 2004;

revision accepted for publication February 16, 2005.) 